\title{
Evidence of the oscillatory magnetic anisotropy in $\mathrm{Ni} / \mathrm{Co} / \mathrm{Ni} / \mathrm{Cu}(100)$
}

\author{
C. Won, ${ }^{1}$ Y. Z. Wu, ${ }^{1}$ N. Kurahashi, ${ }^{1}$ K. T. Law, ${ }^{1, *}$ H. W. Zhao, ${ }^{1,2}$ A. Scholl,${ }^{3}$ A. Doran, ${ }^{3}$ and Z. Q. Qiu ${ }^{1,4}$ \\ ${ }^{1}$ Department of Physics, University of California at Berkeley, Berkeley, California 94720 \\ ${ }^{2}$ International Center for Quantum Structures and Institute of Physics, Chinese Academy of Sciences, Beijing 100080, China \\ ${ }^{3}$ Advanced Light Source, Lawrence Berkeley National Laboratory, Berkeley, California 94720 \\ ${ }^{4}$ Materials Science Division, Lawrence Berkeley National Laboratory, Berkeley, California 94720
}

(Received 10 October 2002; published 30 May 2003)

\begin{abstract}
Magnetic anisotropy of $\mathrm{Ni} / \mathrm{Co} / \mathrm{Ni} / \mathrm{Cu}(100)$ films was studied by the surface magneto-optical Kerr effect and photoemission electron microscopy. The top $\mathrm{Ni}$ and Co films enhance the Curie temperature of the bottom $\mathrm{Ni}$ layer so that the magnetic anisotropy of the bottom Ni layer can be studied in the monolayer (ML) regime. In addition, the top $\mathrm{Ni}$ and Co film thicknesses were chosen to be close to the spin-reorientation transition (SRT) so that the magnetic anisotropy of the bottom Ni layer manifests in the SRT of the whole film. We found that the magnetization direction of the film oscillates as a function of the bottom Ni thickness with a perpendicular magnetic anisotropy appearing at $\sim 2 \mathrm{ML}$ of Ni. This result indicates the existence of the oscillatory magnetic anisotropy of the Ni film in the ultrathin regime.
\end{abstract}

DOI: 10.1103/PhysRevB.67.174425

PACS number(s): 75.70.Cn

\section{INTRODUCTION}

$\mathrm{Ni} / \mathrm{Cu}(100)$ has become one of the prototype systems for the study of magnetic thin films. The interplay between the interfacial effect, magneto-elasticity, and the dipolar magnetic interaction results in many interesting magnetic behaviors in the $\mathrm{Ni} / \mathrm{Cu}(100)$ system. Structurally, $\mathrm{Ni}$ grows pseudomorphically on $\mathrm{Cu}(100)$, up to a critical thickness, with its in-plane lattice extended to that of $\mathrm{Cu}$ and its out-ofplane lattice contracted. ${ }^{1}$ At regions thicker than the critical thickness, strain relaxation occurs by the creation of dislocations, and the Ni lattice gradually relaxes towards its bulk form. ${ }^{2}$ In the pseudomorphic region, the competition of the strain-induced perpendicular magnetoelastic anisotropy against the in-plane interfacial magnetic anisotropy and the shape magnetic anisotropy gives rise to a spin reorientation transition (SRT) from in-plane magnetization below 7 monolayer (ML) Ni to out-of-plane magnetization above $7 \mathrm{ML}$ $\mathrm{Ni}^{3}{ }^{3}$ Much effort has been devoted to understand the Ni SRT around $7 \mathrm{ML}$ of $\mathrm{Ni}$ in terms of the magnetic anisotropy. For example, Farle et al. constructed a phase diagram of the magnetization direction under different conditions, ${ }^{4}$ and showed that the SRT is a continuous rotation of the magnetization direction. ${ }^{5}$ Kuch et al. extended the SRT by covering the Ni film with a thin Co layer to obtain some micromagnetic information. ${ }^{6}$ Dhesi, Dürr, and van der Laan found the in-plane component switching of Ni film grown on stepped $\mathrm{Cu}(100)$ near the SRT. ${ }^{7}$ The effect of $\mathrm{Cu}$ capping layer on the SRT was also studied in details. ${ }^{8}$ In addition to the experimental effort, theoretical calculations were also developed to explain the origins of the magnetic anisotropy. ${ }^{9}$ It was pointed out that the tetragonal distortion of the $\mathrm{Ni}$ film on $\mathrm{Cu}(100)$ is the origin of the strong perpendicular magnetic anisotropy. ${ }^{10}$ It is well known that electronic states in ultrathin films are strongly modulated due to the quantization effect; ${ }^{11}$ thus the magnetic anisotropy of a thin film should be modulated accordingly. Such effect was predicted by layer resolved theoretical calculation in the $\mathrm{Ni} / \mathrm{Cu}(100)$ system. ${ }^{12}$ In particular, the oscillatory magnetic anisotropy has been predicted for ultrathin $\mathrm{Ni}$ films on $\mathrm{Cu}(100)$ which should lead to a perpendicular magnetization at $2 \mathrm{ML}$ of $\mathrm{Ni}^{13}$ Since the magnetic anisotropy has a strong temperature dependence ${ }^{5}$ and that the Curie temperature $\left(T_{C}\right)$ is significantly reduced as the Ni film thickness approaches the ultrathin limit, ${ }^{14}$ it is difficult to study the thickness dependent magnetic anisotropy near $2 \mathrm{ML}$ of $\mathrm{Ni}$. In this paper, we report the study of $\mathrm{Ni} / \mathrm{Co} / \mathrm{Ni} / \mathrm{Cu}(100)$ system. In this system, the bottom Ni layer is in the monolayer regime but its $T_{C}$ is much higher than room temperature due to the presence of the $\mathrm{Co}$ and the top Ni films. The top Ni and Co film thicknesses were chosen to be close to the SRT point so that an oscillatory magnetic anisotropy as a function of the bottom Ni layer thickness can be singled out by studying the SRT of the overall film. We indeed obtained evidence of the oscillatory magnetic anisotropy as a function of the Ni film thickness.

\section{EXPERIMENT}

$\mathrm{A} \mathrm{Cu}(100)$ single-crystal disk was mechanically polished down to $0.25 \mu \mathrm{m}$ diamond paste finish, and then electrochemically polished. ${ }^{15}$ The Cu substrate was then introduced into an ultrahigh vacuum chamber and cleaned by cycles of $\mathrm{Ar}^{+}$sputtering at $2-3 \mathrm{keV}$ and annealing at $\sim 600^{\circ} \mathrm{C}$. Ni and Co films were grown epitaxially onto the $\mathrm{Cu}(100)$ at room substrate temperature by thermally evaporating $\mathrm{Ni}$ and $\mathrm{Co}$ materials contained in $\mathrm{Al}_{2} \mathrm{O}_{3}$ crucibles. The films were characterized by low-energy electron diffraction (LEED), reflection high-energy electron diffraction (RHEED), and Auger electron spectroscopy (AES). The evaporation rate was monitored by a water-cooled quartz balance which was calibrated by RHEED oscillations. For magnetic measurement, several samples were prepared with the Co or Ni layers grown into wedges to permit their thickness control. Surface magneto-optical Kerr effect (SMOKE) measurements were performed in situ at room temperature. Two pairs of electromagnets apply a magnetic field either parallel or perpendicular to the film plane to generate the longitudinal or polar 

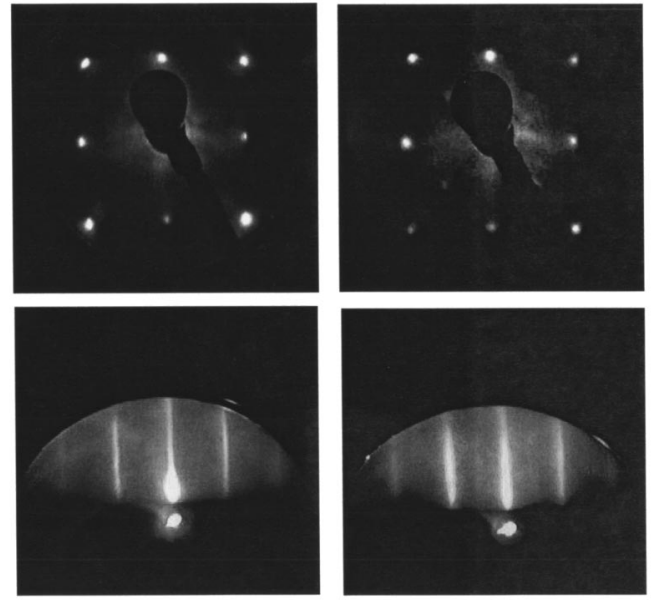

(a)

(b)

FIG. 1. LEED and RHEED patterns of (a) $\mathrm{Cu}(100)$ and (b) $\mathrm{Ni}(5$ $\mathrm{ML}) / \mathrm{Co}(2 \mathrm{ML}) / \mathrm{Ni}(5 \mathrm{ML}) / \mathrm{Cu}(100)$.

magnetic hysteresis loops without changing the sample position. The SMOKE incident light is a He-Ne laser and is focused on to the sample surface with an optic lens. The incident angle is fixed at $\sim 45^{\circ}$ relative to the normal direction of the surface. After reflection, the beam intensity is measured by a photodiode behind a linear polarizer which is set at $\sim 1^{\circ}$ from extinction. The sample for photoemission electron microscopy (PEEM) measurement was capped with a $20 \AA \mathrm{Cu}$ protective layer and transferred into the PEEM chamber at beamline 7.3.1.1 of the Advanced Light Source (ALS) at Lawrence Berkeley National Laboratory (LBNL). The starting positions of the $\mathrm{Ni}$ and $\mathrm{Co}$ wedges were determined using the $2 p$ core levels, and the magnetic domain images were obtained by taking the ratio of $L_{3}$ and $L_{2}$ edges ${ }^{16}$ utilizing the effect of $\mathrm{x}$-ray magnetic circular dichroism (XMCD).

\section{RESULTS}

\section{A. Structural characterization}

Figure 1 shows typical LEED and RHEED patterns of the $\mathrm{Cu}(100)$ substrate and the $\mathrm{Ni} / \mathrm{Co} / \mathrm{Ni}$ film grown on top of the $\mathrm{Cu}(100)$. There is no obvious change in the diffraction patterns after the film growth, suggesting an epitaxial growth nature of this system. To further confirm this, RHEED intensity was monitored during film growth. The presence of the RHEED oscillations (Fig. 2) confirms that this system indeed has a nearly layer-by-layer growth mode. For the $\mathrm{Ni} / \mathrm{Cu}(100)$ system, there exists a critical thickness above which the strain relaxation occurs. However, the value of the critical thickness depends on the quality of the film and is not well defined. Ha et al. studied $\mathrm{Cu} / \mathrm{Ni} / \mathrm{Cu} / \mathrm{Si}(100)$ in the Ni thickness range of $30-2000 \AA .{ }^{17}$ However, the value of the $\mathrm{Ni}$ critical thickness depends on the model fitting. Their experimental data suggest that Ni films below $30 \AA$ have a strain very close to that in the pseudomorphic region (dislocation spacing greater than $200 \AA$ ). For the Ni film grown on a single-crystal $\mathrm{Cu}(100)$ substrate, a LEED $I-V$ study shows little change up to $22 \mathrm{ML}$ of $\mathrm{Ni}^{18}{ }^{18}$ These results suggest that strain relaxation does not have a significant effect on $\mathrm{Ni}$ films below 20-30 ML. In fact, the second SRT due to strain relaxation occurs at $\sim 135 \AA$ of Ni thickness. We will come back to this point later when discussing the magnetic properties.

\section{B. $\mathrm{Ni} / \mathrm{Co} / \mathrm{Cu}(\mathbf{1 0 0})$}

We first studied the $\mathrm{Ni} / \mathrm{Co} / \mathrm{Cu}(100)$ system. Double wedges of $\mathrm{Ni}$ and $\mathrm{Co}$ were grown on $\mathrm{Cu}(100)$ and measured in situ by SMOKE. Polar hysteresis loops of the films were taken at room temperature. Figure 3 shows the remanent polar signal, which is proportional to the perpendicular component of the magnetization $M_{\perp}$, as a function of the Ni film thickness with 0,1 , and 2 ML Co. The perpendicular component of the magnetization remains zero in thin film regime and develops above a critical thickness. The onset of $M_{\perp}$

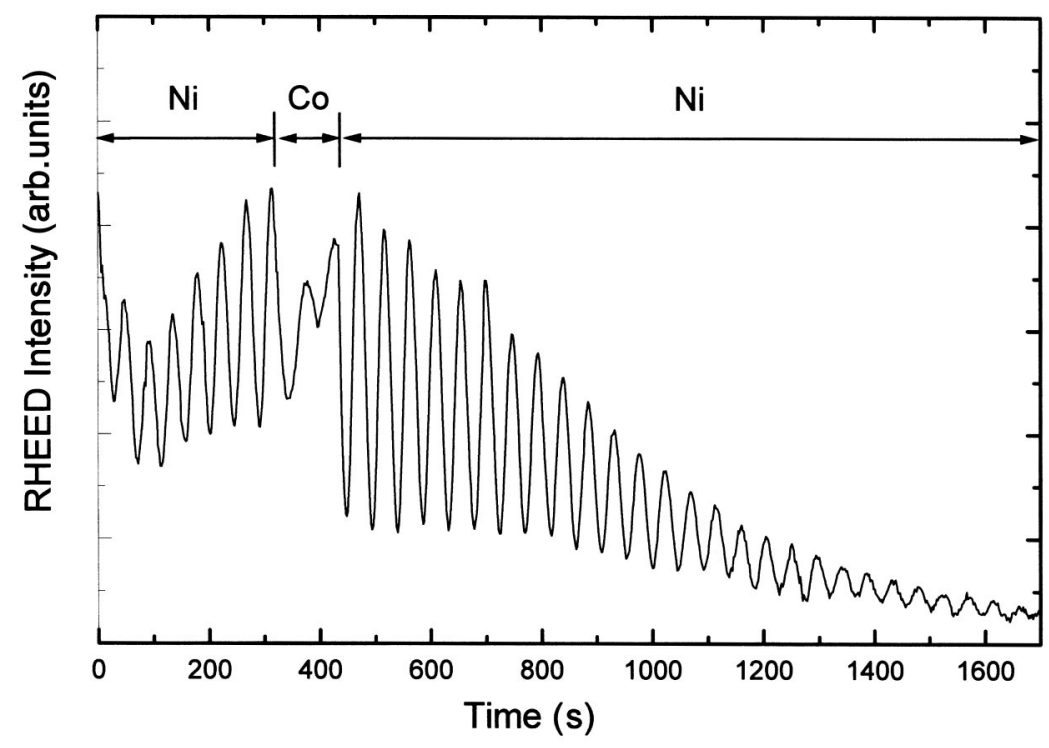

FIG. 2. RHEED intensity versus growth time. The presence of RHEED oscillations suggests an epitaxial growth nature of the $\mathrm{Ni} / \mathrm{Co} / \mathrm{Ni} / \mathrm{Cu}(100)$. 


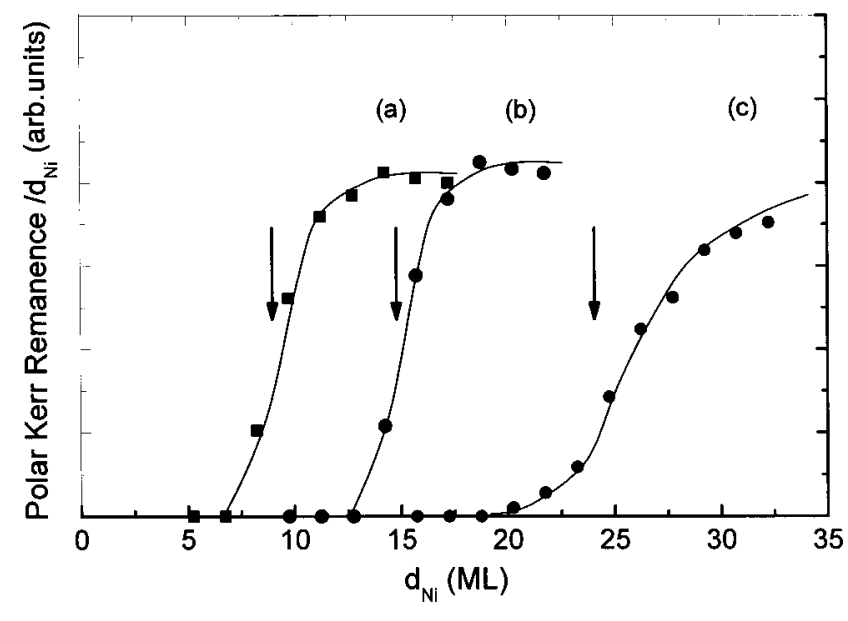

FIG. 3. Polar magnetic remanence of the $\mathrm{Ni} / \mathrm{Co} / \mathrm{Cu}(100)$ as a function of the Ni film thickness. The arrow defines the SRT thickness. The solid lines are guide to eye.

signatures the in-plane to out-of-plane SRT of the Ni films. We define the SRT thickness as the thickness at which the polar Kerr signal starts to develop $(\sim 4 \%$ Kerr signal, indicated by arrows in Fig. 3). The choice of $4 \%$ is not essential but is just for operational convenience. Obviously, the presence of a Co layer increases the Ni SRT thickness. The SRT thickness was determined systematically from the $\mathrm{Ni} / \mathrm{Co} /$ $\mathrm{Cu}(100)$ double-wedged sample (Fig. 4). The critical thickness plotted in the $d_{\mathrm{Ni}^{-}} d_{\mathrm{Co}}$ plane can be roughly divided into two segments $\left(d_{\mathrm{Co}}<1 \mathrm{ML}\right.$ and $\left.d_{\mathrm{Co}}>1 \mathrm{ML}\right)$, each behaving linearly. For $d_{\mathrm{Co}}>1 \mathrm{ML}$, the linear coefficient is $d_{\mathrm{Ni}} / d_{\mathrm{Co}}$ $=10.4$, in agreement with Ref. 6 . For $d_{\mathrm{Co}}<1 \mathrm{ML}$, the linear coefficient is $d_{\mathrm{Ni}} / d_{\mathrm{Co}}=5.5$, less than that of $d_{\mathrm{Co}}>1 \mathrm{ML}$.

The linear dependence in Fig. 4 can be understood in terms of the anisotropy competition between the $\mathrm{Ni}$ and $\mathrm{Co}$

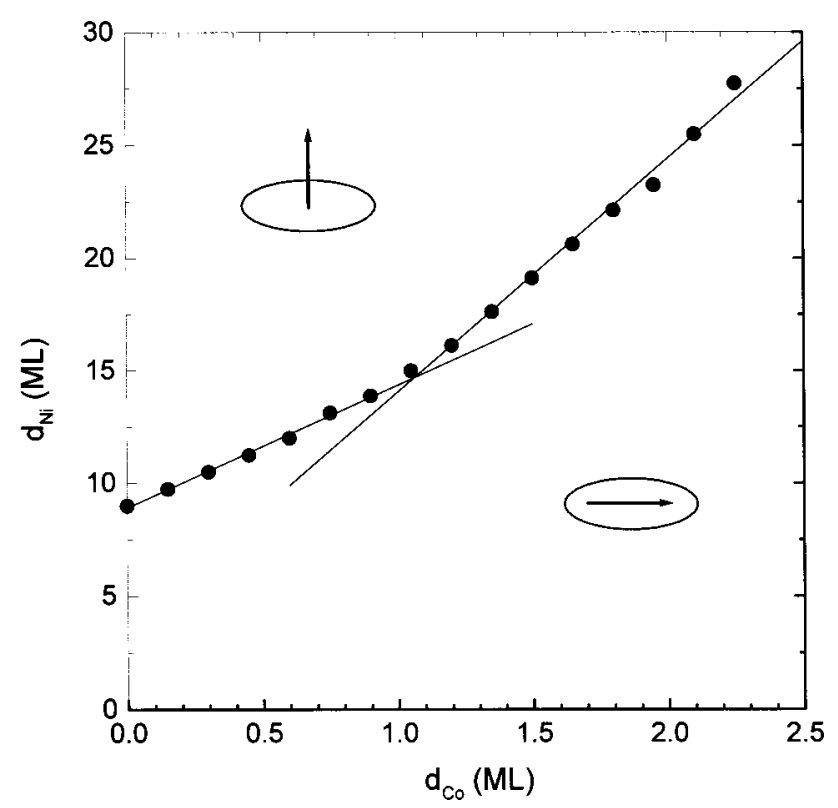

FIG. 4. SRT thickness in the $d_{\mathrm{Ni}^{-}} d_{\mathrm{Co}}$ plane. Linear relations between $d_{\mathrm{Ni}}$ and $d_{\mathrm{Co}}$ can be obtained for $d_{\mathrm{Co}}<1 \mathrm{ML}$ and $d_{\mathrm{Co}}>1 \mathrm{ML}$. films. Assuming that the strong Ni-Co exchange coupling lines up the $\mathrm{Ni}$ and Co magnetizations in the same direction, the area uniaxial magnetic anisotropy of the $\mathrm{Ni} / \mathrm{Co} / \mathrm{Cu}(100)$ film for $d_{\mathrm{Co}}>1 \mathrm{ML}$ is

$$
\begin{aligned}
E= & \left(-2 \pi M_{\mathrm{Ni}}^{2}+K_{V}^{\mathrm{Ni}}\right) d_{\mathrm{Ni}}+\left(-2 \pi M_{\mathrm{Co}}^{2}+K_{V}^{\mathrm{Co}}\right) d_{\mathrm{Co}}+K_{S}^{\mathrm{Ni}-\mathrm{vac}} \\
& +K_{S}^{\mathrm{Ni}-\mathrm{Co}}+K_{S}^{\mathrm{Co}-\mathrm{Cu}} .
\end{aligned}
$$

Here the $V$ and $S$ subscripts of $K$ denote the volume and surface magnetic anisotropies, and the superscript of $K$ states where the anisotropy comes from. Then a linear dependence is expected between $d_{\mathrm{Ni}}$ and $d_{\mathrm{Co}}$ for the SRT $(E=0)$.

For $d_{\mathrm{Co}}<1 \mathrm{ML}$, the Co film should form 1-ML islands with the top Ni film contacting the $\mathrm{Cu}$ substrate in the pinhole region. Thus the area uniaxial magnetic anisotropy under this condition should be

$$
\begin{aligned}
E= & \left(-2 \pi M_{\mathrm{Ni}}^{2}+K_{V}^{\mathrm{Ni}}\right) d_{\mathrm{Ni}}+\left(-2 \pi M_{\mathrm{Co}}^{2}+K_{V}^{\mathrm{Co}}\right) d_{\mathrm{Co}}+K_{S}^{\mathrm{Ni}-\mathrm{vac}} \\
& +\left(K_{S}^{\mathrm{Ni}-\mathrm{Co}}+K_{S}^{\mathrm{Co}-\mathrm{Cu}}\right) d_{\mathrm{Co}} /(1 \mathrm{ML}) \\
& +K_{S}^{\mathrm{Ni}-\mathrm{Cu}}\left(1-d_{\mathrm{Co}}\right) /(1 \mathrm{ML})
\end{aligned}
$$

Here $d$ is in units of ML, and thus $d_{\mathrm{Co}}$ and $1-d_{\mathrm{Co}}$ describe the fraction of Co islands and pinholes, respectively. Equation (2) defines a straight line in the $d_{\mathrm{Ni}^{-}} d_{\mathrm{Co}}$ plane for the SRT but with a different slope than that defined by Eq. (1). The crossover between Eqs. (1) and (2) should occur at $d_{\text {Co }}$ $\sim 1 \mathrm{ML}$, which agrees roughly with the experimental result in Fig. 4. The less steep slope at $d_{\mathrm{Co}}<1 \mathrm{ML}$ shows that $K_{S}^{\mathrm{Ni}-\mathrm{Co}}+K_{S}^{\mathrm{Co}-\mathrm{Cu}}>K_{S}^{\mathrm{Ni}-\mathrm{Cu}}$. One could attempt to do some quantitative estimate of the magnetic anisotropy from the slopes of Eqs. (1) and (2), but a large error could be generated using this method. ${ }^{18}$ Another interesting feature is that there is no obvious deviation from the linear behavior in the $d_{\mathrm{Ni}^{-}}-d_{\mathrm{Co}}$ plane up to $d_{\mathrm{Co}}=2 \mathrm{ML}$ and $d_{\mathrm{Ni}}=25 \mathrm{ML}$. Noting that strain relaxation changes the magnetic anisotropy, the linear behavior in Fig. 4 indicates that strain relaxation does not play a significant role in the thickness range studied.

\section{C. $\mathrm{Ni} / \mathrm{Co} / \mathrm{Ni} / \mathrm{Cu}(\mathbf{1 0 0})$}

The $\mathrm{Ni} / \mathrm{Co} / \mathrm{Ni} / \mathrm{Cu}(100)$ sample was grown with the two $\mathrm{Ni}$ films as wedges and the Co film at fixed thickness. SRT thickness was determined for several samples with different Co thicknesses. As shown in Fig. 5, the results for 0.7, 1.4, and 1.8 ML Co samples are from the SMOKE measurements, and the result for 1.1 ML Co is from the PEEM measurement. The SRT lines determine the relation of the top and bottom Ni thicknesses $\left(d_{t}\right.$ and $\left.d_{b}\right)$ that give rise to an overall zero magnetic anisotropy. By increasing the bottom Ni layer thickness $\left(d_{b}\right)$, the top layer's critical thickness $\left(d_{t}\right)$ first decreases to reach a minimum value at $d_{b} \sim 2 \mathrm{ML}$, then increases to a peak, and finally decreases by further increasing $d_{b}$. The dependence of $d_{t}$ on $d_{b}$ obviously exhibits an oscillatory behavior. This oscillation can be seen clearly in the PEEM and SMOKE measurements on samples of fixed top Ni layer thickness. 


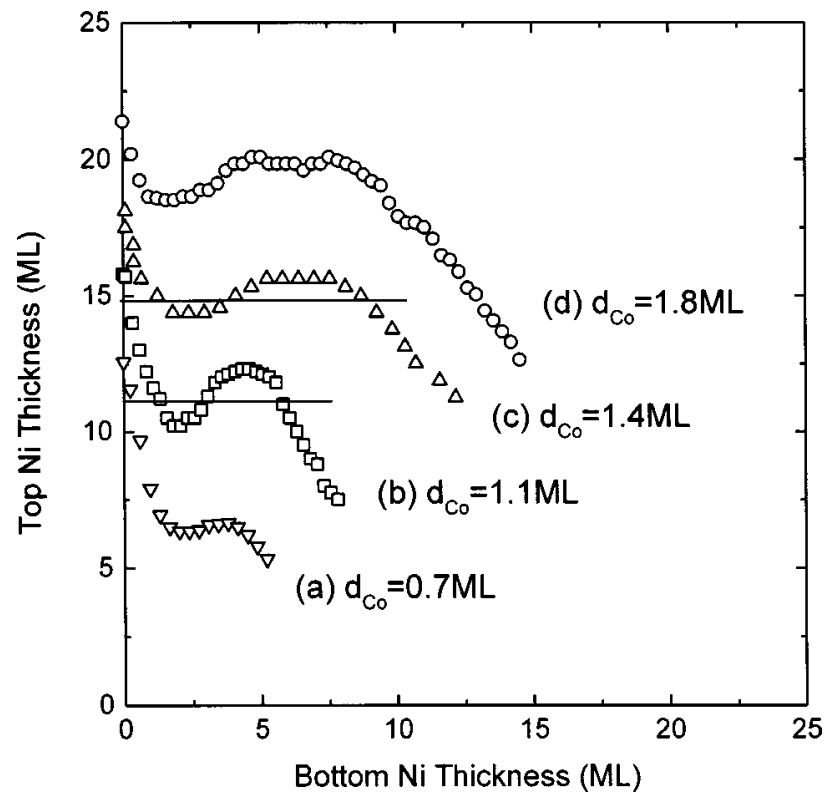

FIG. 5. SRT thickness in the $d_{t}-d_{b}$ plane for sample of $\mathrm{Ni}\left(d_{t}\right) / \mathrm{Co} / \mathrm{Ni}\left(d_{b}\right) / \mathrm{Cu}(100)$.

Figure 6 shows a series of PEEM images of the sample, $\mathrm{Ni}(11 \mathrm{ML}) / \mathrm{Co}(1.1 \mathrm{ML}) / \mathrm{Ni}\left(d_{b}\right) / \mathrm{Cu}(100)$ [along the horizontal line of Fig. 5(b)]. Before taking the PEEM measurements, the sample was magnetized by an external magnetic field to wipe out perpendicular magnetic domains so that the in-plane and out-of-plane magnetizations can be distinguished clearly in the PEEM images. Due to the strong Ni-Co magnetic coupling, we did not observe any difference between the $\mathrm{Ni}$ and $\mathrm{Co}$ magnetic domains so that we will only show the Ni domain images in this paper. With the increase of the bottom $\mathrm{Ni}$ layer thickness, the film exhibits in-plane magnetic domains below $1 \mathrm{ML}$ of the bottom Ni layer, then an in-plane to out-of-plane transition at $d_{b}$ $\sim 1.3 \mathrm{ML}$, another out-of-plane to in-plane transition at $d_{b}$ $\sim 3.0 \mathrm{ML}$, and finally an in-plane to out-of-plane transition at $d_{b} \sim 5.8 \mathrm{ML}$. The parallel-to-perpendicular-to-parallel-toperpendicular SRT's are clearly seen. Similar behavior was also observed by the SMOKE measurements.

Figure 7 shows the polar and longitudinal SMOKE remanences of $\mathrm{Ni}(14.7 \mathrm{ML}) / \mathrm{Co}(1.4 \mathrm{ML}) / \mathrm{Ni}\left(d_{b}\right) / \mathrm{Cu}(100)$ as a function of the bottom Ni layer thickness [along the horizontal line of Fig. 5(c)]. The polar signal has been rescaled and shifted in order to plot it in the same graph with the longitudinal signal. Both the polar and longitudinal remanences oscillate with $d_{b}$ in an out-of-phase manner, showing the parallel-to-perpendicular-to-parallel-to-perpendicular SRT's as a function of the bottom Ni layer thickness.

\section{DISCUSSION}

We now discuss the origins of the oscillatory behavior in Figs. 6 and 7 . The $\mathrm{Ni}\left(d_{t}\right) / \mathrm{Co}$ layers in the $\mathrm{Ni}\left(d_{t}\right) / \mathrm{Co} / \mathrm{Ni}\left(d_{b}\right) / \mathrm{Cu}(100)$ system play two roles. First, the ferromagnetic order of the $\mathrm{Ni}\left(d_{t}\right)$ /Co layers greatly enhance the Curie temperature of the Ni bottom layer as compared to the $\mathrm{Ni} / \mathrm{Cu}(100)$ system so that we can ignore the temperature effect. Second, the top Ni layer thickness is near the SRT critical thickness of the $\mathrm{Ni} / \mathrm{Co} / \mathrm{Cu}(100)$ system so that the magnetic anisotropy of the $\mathrm{Ni}\left(d_{t}\right) / \mathrm{Co}$ layers in the $\mathrm{Ni}\left(d_{t}\right) / \mathrm{Co} / \mathrm{Ni}\left(d_{b}\right) / \mathrm{Cu}(100)$ system should be close to zero. Then the SRT of the $\mathrm{Ni}\left(d_{t}\right) / \mathrm{Co} / \mathrm{Ni}\left(d_{b}\right) / \mathrm{Cu}(100)$ system shown in Figs. 6 and 7 should reflect the magnetic anisotropy change due to the bottom $\mathrm{Ni}$ layer. The parallel-toperpendicular SRT at thicker bottom Ni layer $\left(d_{b} \sim 6 \mathrm{ML}\right)$ for Fig. 6 and $d_{b} \sim 10 \mathrm{ML}$ for Fig. 7) corresponds to the SRT of the $\mathrm{Ni} / \mathrm{Cu}(100)$ at $\sim 7 \mathrm{ML}$ of $\mathrm{Ni}$. However, the perpendicular magnetization around 2-3 ML bottom Ni layer is a new observation that was not reported in the literature. Since the oscillatory behavior in Figs. 6 and 7 is present throughout the Co thickness range studied $\left(0.7 \mathrm{ML}<d_{\mathrm{Co}}<1.8 \mathrm{ML}\right)$ and the local minimum of $d_{t}$ is always around $d_{b} \sim 2 \mathrm{ML}$, we can rule out the effect of pinholes in the Co layer.

Pinholes in the bottom Ni layer are expected to exist for $d_{b}<1 \mathrm{ML}$. In such a case, the Co film should be in contact with the $\mathrm{Cu}$ substrate. This effect may account for the rapid decrease of the top Ni thickness $\left(d_{t}\right)$ in Fig. 5 in the region of $d_{b}<1 \mathrm{ML}$, but it does not explain why $d_{t}$ increases again $\left(d_{b}>2 \mathrm{ML}\right)$ after the bottom Ni layer separates the Co and $\mathrm{Cu}$. Thus we can also rule out the effect of pinholes from the bottom Ni layer. Therefore the oscillations of the SRT critical thickness in Fig. 5 and the oscillations of the magnetization direction in Figs. 6 and 7 show the existence of the oscillatory magnetic anisotropy in $\mathrm{Ni} / \mathrm{Co} / \mathrm{Ni} / \mathrm{Cu}(100)$. Since only $d_{b}$ varies in Figs. 6 and 7, the oscillatory magnetic anisotropy should be due to the bottom Ni layer. Because of the sensitive dependence of the Ni magnetic anisotropy on its tetragonal distortion, ${ }^{10}$ we cannot further isolate the magnetic anisotropy of the $\mathrm{Ni}$ bottom layer from the overall structure, i.e., we do not know if the perpendicular magnetic anisotropy around $d_{b} \sim 2 \mathrm{ML}$ in the $\mathrm{Ni} / \mathrm{Co} / \mathrm{Ni} / \mathrm{Cu}(100)$ will be a universal behavior for $\mathrm{Ni}$ in any other structures.

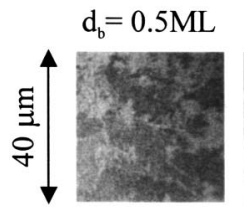

(a)

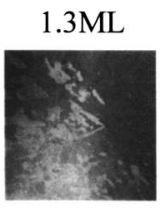

(b)

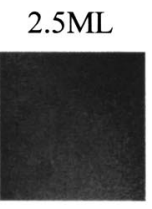

(c)

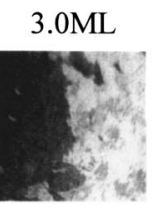

(d)

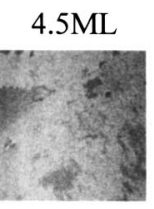

(e)

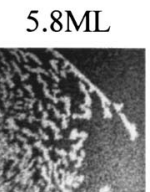

(f)
7.0ML

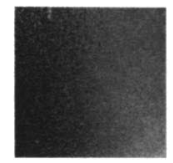

(g)

FIG. 6. PEEM images from the sample of $\mathrm{Ni}(11 \mathrm{ML}) / \mathrm{Co}(1.1 \mathrm{ML}) / \mathrm{Ni}\left(d_{b}\right) / \mathrm{Cu}(100)$. (a) and (e) show the in-plane magnetization. (c) and (g) show the perpendicular magnetization. (b), (d), and (f) show the SRT regions. The oscillations of the magnetization direction with $d_{b}$ indicate the existence of the oscillatory magnetic anisotropy. 


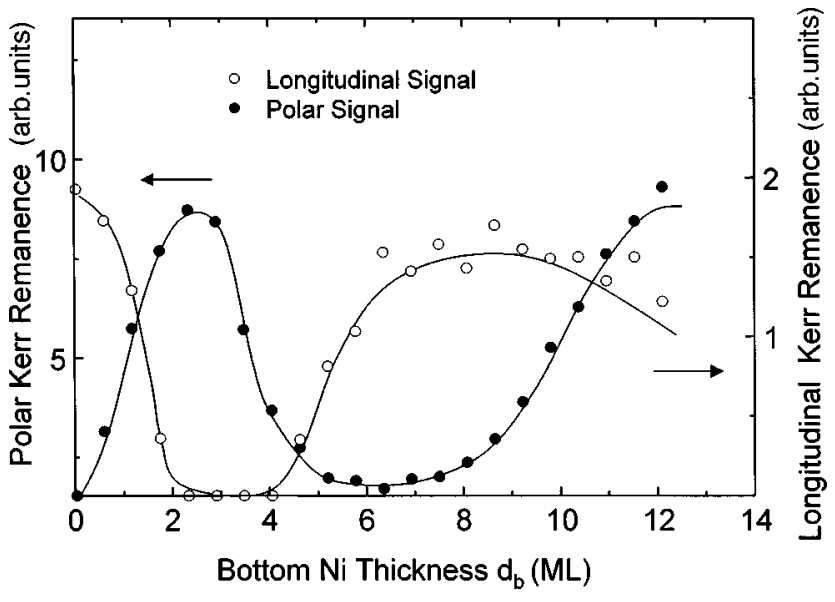

FIG. 7. Polar and longitudinal remanences of $\mathrm{Ni}(14.7 \mathrm{ML}) / \mathrm{Co}(1.4 \mathrm{ML}) / \mathrm{Ni}\left(d_{b}\right) / \mathrm{Cu}(100)$ as a function of the bottom Ni layer thickness. The solid lines are guide to eye. The oscillations with $d_{b}$ show the existence of the oscillatory magnetic anisotropy.

As pointed out in the Introduction, the magnetic anisotropy of fct $\mathrm{Ni}$ film on $\mathrm{Cu}(100)$ depends strongly on the lattice distortion and the electronic states. These two effects are both present in thin films, making it difficult to separate them. However, it would be constructive to discuss them in terms of existing experimental and theoretical works. First, we would like to discuss the effect of the lattice distortion. Since the Ni lattice contraction in the vertical direction of the film originates its perpendicular magnetoelastic anisotropy, a greater lattice contraction of the bottom Ni layer at $\sim 2 \mathrm{ML}$ in $\mathrm{Ni} / \mathrm{Co} / \mathrm{Ni} / \mathrm{Cu}(100)$ could explain our experimental result. For the $\mathrm{Ni} / \mathrm{Co} / \mathrm{Ni} / \mathrm{Cu}(100)$ system, no detailed structural characterization is available in the literature. For the $\mathrm{Ni}$ / $\mathrm{Cu}(100)$ system, LEED $I-V$ study shows no obvious different interlayer spacing around $2 \mathrm{ML}$ Ni film. ${ }^{19}$ Magnetic measurement on $\mathrm{Ni}(1.6 \mathrm{ML}) / \mathrm{Cu}(100)$ also shows an in-plane magnetization. ${ }^{20}$ However, because of the low Curie temperature of the $\mathrm{Ni}(1.6 \mathrm{ML}) / \mathrm{Cu}(100)$ and the strong dependence of the magnetic anisotropy on $T / T_{C}$, the observed in-plane magnetic anisotropy in $\mathrm{Ni}(1.6 \mathrm{ML}) / \mathrm{Cu}(100)$ may not represent its value far below $T_{C}$. Therefore, we cannot rule out the lattice distortion effect in our experiment without further experiments on the lattice contraction around $2 \mathrm{ML}$ of the bottom $\mathrm{Ni}$ in $\mathrm{Ni} / \mathrm{Co} / \mathrm{Ni} / \mathrm{Cu}(100)$ and a retesting of the magnetization direction of $\mathrm{Ni} / \mathrm{Cu}(100)$ around $2 \mathrm{ML} \mathrm{Ni}$ at a very low temperature. Second, we would like to discuss the effect of the electronic state in our experiment. In ultrathin films, the interfaces reflect electron waves to form quantum well (QW) states. The energy levels of the QW states depend on the film thickness and the interfacial properties. The modification of the electronic states due to the QW states should, in principle, modify the magnetic anisotropy of a magnetic thin film to result in an oscillatory behavior. Some theoretical results may shed light on this effect. Uiberacker et al. performed layer-resolved calculation on the $\mathrm{Ni}$ / $\mathrm{Cu}(100)$ system and showed that the magnetic anisotropy exhibits oscillations with the Ni thickness and has a perpendicular magnetization at $2 \mathrm{ML}$ of $\mathrm{Ni}$ in a wide range of the strain $(0$ to $-5.5 \%){ }^{13}$ However, it is difficult to make a direct comparison between this theoretical work and our experiment because our experimental system is not a single $\mathrm{Ni}$ film on $\mathrm{Cu}(100)$. Another qualitative explanation is the quantum interference between different layers. In a multiplayer system, multielectron reflections could generate quantum interference effect. ${ }^{21}$ The presence of the Ni, Co, and Ni layers in the $\mathrm{Ni} / \mathrm{Co} / \mathrm{Ni} / \mathrm{Cu}(100)$ can be regarded as a multi-QW system. Thus QW interference between different layers should affect the electronic states of the system to modulate the magnetic anisotropy. Future theoretical works are suggested to explore such possibility.

\section{SUMMARY}

$\mathrm{Ni} / \mathrm{Co} / \mathrm{Ni} / \mathrm{Cu}(100)$ films are grown and studied by SMOKE and PEEM. The critical thickness of the spin reorientation transition was determined for fixed Co film thickness. We found that the SRT thickness and the magnetization direction oscillate as a function of the bottom Ni film thickness. In particular, a perpendicular magnetic anisotropy was observed at $\sim 2 \mathrm{ML}$ of the bottom Ni layer. These results suggest the existence of the oscillatory magnetic anisotropy of the Ni film in the ultrathin regime.

\section{ACKNOWLEDGMENT}

This work was funded in part by the National Science Foundation under Contract No. DMR-0110034, the U.S. Department of Energy under Contract No. DE-AC0376SF00098, and the ICQS of the Chinese Academy of Science.
*Present address: Department of Physics, Hong Kong University of Science and Technology

${ }^{1}$ S. Müller, B. Schulz, G. Kostka, M. Farle, K. Heinz, and K. Baberschke, Surf. Sci. 364, 235 (1996).

${ }^{2}$ K. Ha, M. Ciria, R. C. O'Handley, P. W. Stephens, and S. Pagola, Phys. Rev. B 60, 13780 (1999).

${ }^{3}$ B. Schulz and K. Baberschke, Phys. Rev. B 50, 13467 (1994).

${ }^{4}$ M. Farle, B. Mirwald-Schulz, A. N. Anisimov, W. Platow, and K. Baberschke, Phys. Rev. B 55, 3708 (1997).

${ }^{5}$ M. Farle, W. Platow, A. N. Anisimov, P. Poulopoulos, and K. Baberschke, Phys. Rev. B 56, 5100 (1997).
${ }^{6}$ W. Kuch, J. Gilles, S. S. Kang, S. Imada, S. Suga, and J. Kirschner, Phys. Rev. B 62, 3824 (2000).

${ }^{7}$ S. S. Dhesi, H. A. Dürr, and G. van der Laan, Phys. Rev. B 59, 8408 (1999).

${ }^{8}$ H. W. Zhao, Y. Z. Wu, C. Won, F. Toyoma, and Z. Q. Qiu, Phys. Rev. B 66, 104402 (2001).

${ }^{9}$ D. Wang, R. Wu, and A. J. Freeman, Phys. Rev. Lett. 70, 869 (1993).

${ }^{10}$ O. Hjortstam, K. Baberschke, J. M. Wills, B. Johansson, and O. Eriksson, Phys. Rev. B 55, 15026 (1997).

${ }^{11}$ R. K. Kawakami, E. Rotenberg, Hyuk J. Choi, Ernesto J. 
Escorcia-Aparicio, M. O. Bowen, J. H. Wolfe, E. Arenholtz, Z. Zhang, N. V. Smith, and Z. Q. Qiu, Nature (London) 398, 132 (1999)

${ }^{12}$ J. Henk, A. M. N. Niklasson, and B. Johansson, Phys. Rev. B 59, 9332 (1999).

${ }^{13}$ C. Uiberacker, J. Zabloudil, P. Weinberger, L. Szunyogh, and C. Sommers, Phys. Rev. Lett. 82, 1289 (1999).

${ }^{14}$ Renjun Zhang and Roy F. Willis, Phys. Rev. Lett. 86, 2665 (2001).

${ }^{15}$ R. K. Kawakami, M. O. Bowen, H. J. Choi, E. J. EscorciaAparicio, and Z. Q. Qiu, Phys. Rev. B 58, R5924 (1998).

${ }^{16}$ F. Nolting, A. Scholl, J. Stöhr, J. W. Seo, J. Fompeyrine, H. Siegwart, J.-P. Loquet, S. Anders, J. Lüning, E. E. Fullerton, M. F.
Toney, M. R. Scheinfein, and H. A. Padmore, Nature (London) 405, 767 (2000).

${ }^{17}$ Gabriel Bochi, C. A. Ballentine, H. E. Inglefield, C. V. Thompson, and R. C. O'Handley, Phys. Rev. B 53, 1729 (1996).

${ }^{18}$ M. Zheng, J. Shen, P. Ohresser, Ch. V. Mohan, M. Klaua, J. Barthel, and J. Kirschner, J. Appl. Phys. 85, 5060 (1999).

${ }^{19}$ W. Platow, U. Bvensiepen, P. Poulopoulos, M. Farle, K. Baberschke, L. Hammer, S. Walter, S. Müller, and K. Heinz, Phys. Rev. B 59, 12641 (1999).

${ }^{20}$ M. Tischer, D. Arvanitis, T. Yokoyama, T. Lederer, L. Troger, and K. Baberschke, Surf. Sci. 307, 1096 (1994).

${ }^{21}$ R. K. Kawakami, E. Rotenberg, E. J. Escorcia-Aparicio, H. J. Choi, T. R. Cummins, J. G. Tobin, N. V. Smith, and Z. Q. Qiu, Phys. Rev. Lett. 80, 1754 (1998). 